\title{
NANOENCAPSULATION: A PROMISING STRATEGY FOR BIOMEDICAL APPLICATIONS OF FERULIC ACID
}

\author{
Parichehr Hassanzadeh $^{1^{*}}$, Fatemeh Atyabi ${ }^{1,2}$, and Rassoul Dinarvand ${ }^{1,2}$ \\ ${ }^{1}$ Nanotechnology Research Center, Faculty of Pharmacy, Tehran University of Medical Sciences, Tehran, Iran \\ ${ }^{2}$ Department of Pharmaceutics, Faculty of Pharmacy, Tehran University of Medical Sciences, Teheran, Iran
}

\section{ABSTRACT}

Over the last decades, nutraceuticals and natural products have been the focus of intense research. Ferulic acid, an active phenolic constituent of many plant species with a wide-spectrum of pharmacological properties (e.g. effects against the oxidative stress, thrombosis, diabetes, aging, and neurotoxicity) has attracted a growing interest in the area of biomedical research and clinical practice. Ferulic acid is able to affect a variety of signaling pathways including those of the brain suggesting the influential role of this phytochemical on the neuroinflammatory and neurodegenerative processes as well as the neuronal survival and plasticity. However, poor solubility and physiochemical instability may negatively affect the efficiency of this ubiquitous nutraceutical. In recent years, tremendous research efforts in nanotechnology have provided highly advanced drug delivery systems for improved pharmacological profile of the therapeutic agents, targeted drug delivery, controlled release, and reduced side effects. The present review highlights the therapeutic significance of ferulic acid and the research progress regarding the development of ferulic acid-based nanoformulations. Biomed Rev 2017; 28: 22-30.

Keywords: nanotechnology, nutraceuticals, phenolic compound, phytochemical, drug delivery

\section{INTRODUCTION}

The therapeutic potentials of the naturally occurring phenolic compounds against a variety of disorders have been the focus of intense research (1). Ferulic acid (FA) [(E)-3-(4-hydroxy3-methoxy-phenyl)prop-2-enoic acid] (Fig. 1), one of the most abundant phenolic compounds in plants and foods, has attracted a growing interest due to its wide spectrum of pharmacological activities such as the effects against the inflammation, oxidative stress, aging, diabetes, and neurological disorders.

This multifunctional nutraceutical exhibits free radical scavenging, antimicrobial, and hepatoprotective activities, and protective effects against the UV and ionizing radiation, and reduces triglycerides and cholesterol (2-8). Because of

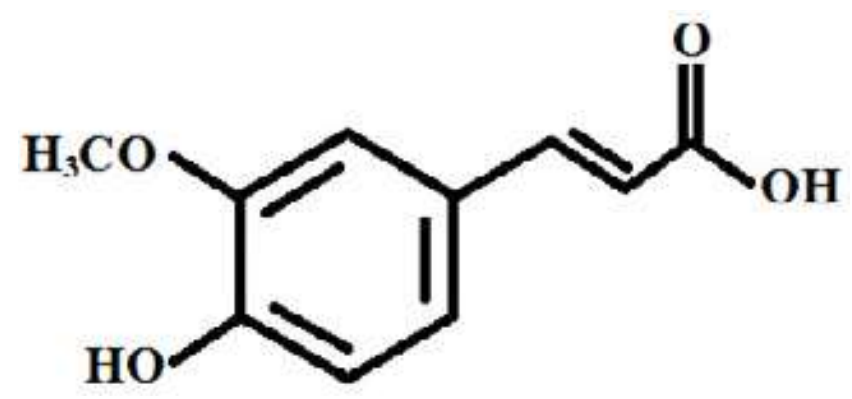

Figure 1. The chemical structure of ferulic acid.

the stimulation of cytoprotective enzymes, scavenging of reactive oxygen species (ROS), and inactivation of certain proteins, FA may be used as an anticancer agent. It has also been suggested that FA reduces the side effects of radio- or

\footnotetext{
* Received 20 November 2017, accepted 2 December 2017. Correspondence and reprint request to Dr Parichehr Hassanzadeh, Nanotechnology Research Center, Faculty of Pharmacy, Tehran University of Medical Sciences, Tehran, Iran

Tel.: +98 21 66959095, Fax: +98 21 66581558, Cell phone: +98 912 1887745,

E-mail: p-hassanzadeh@razi.tums.ac.ir
} 
chemotherapy through the enhancement of the innate immune defense $(9,10)$. Moreover, FA prevents atherogenesis, platelet aggregation, and thrombus formation (11), hence, it might be useful against the ischemic disorders. Ferulic acid has also been used as an ingredient in a variety of drugs, nutraceuticals, and cosmetics $(12,13)$. This ubiquitous phenolic compound through the stimulation of platelet-derived growth factor and vascular endothelial growth factor has shown angiogenic effects both in vitro and in vivo. It appears that mitogen-activated protein kinase (MAPK) and phosphatidylinositol 3-kinase (PI3K) pathways are involved in the angiogenic activity of FA (14). Recently, implication of PI3K signaling pathway in the protective effects of FA against the ischemic neural injuries has been reported (15). Chronic treatment with FA results in the antidepressant-like effects by modulation of the nitrosative/oxidative stress and activities of MAPK/extracellular signal-regulated kinase, $\mathrm{Ca}^{2+} /$ calmodulin-dependent protein kinase II, protein kinase $\mathrm{C}$, protein kinase $\mathrm{A}$, and PI3K pathway $(16,17)$. Based on the neuroprotective effects of neurotrophins against the neuropsychiatric disorders (18-20), the effect of FA on the production of nerve growth factor (NGF), the first neurotrophin discovered by Rita LeviMontalcini (21), which regulates the cognitive function and stress (22) and is involved in the mechanisms of action of a wide variety of psychotropic agents $(19,20,23-28)$ has been investigated. It has been shown that FA is able to stimulate the production of NGF in the brain regions implicated in the modulation of emotions (29). In the frontal cortex, FAinduced enhancement of NGF (29) might be of therapeutic significance as stress results in the reduced cell proliferation (30). FA by significant elevation of NGF in the hippocampus (29) may be involved in the hippocampal neurogenesis and improved cognitive function as NGF is critically implicated in the hippocampal neurogenesis and plasticity (31). According to the facilitatory role of NGF on the cholinergic neurotransmission between the amygdala and nucleus basalis (32), FA-induced enhancement of NGF protein level in the amygdala (29) may result in the improved cognitive performance. Based on the important role of NGF in the development of the olfactory system (33), FA by increasing NGF content in the olfactory bulbs (29), may affect the function of this system. In an attempt to identify a mechanism by which FA affects brain NGF protein levels, the implication of the endocannabinoid system (eCBs) which its interaction with neurotrophic factors, regulatory role in the emotional states, and treatment of neuropsychiatric diseases are well-documented (34-38), has been shown (29). Noteworthy, endocannabinoids and NGF were increased in the same brain regions after treatment with FA (29) suggesting the existence of a balance between the NGF and eCBs for FA activity. Despite the promising therapeutic effects, poor solubility and bioavailability and the physiochemical instability of FA (39) may negatively affect its efficiency. In order to improve the pharmacological profile of FA including its cellular uptake, a variety of FA-loaded nanoformulations have been developed which will be described as follows.

\section{NANOFORMULATIONS OF FERULIC ACID}

Over the last decades, outstanding breakthroughs in nanotechnology have led to the development of more innovative therapeutic agents. Nanoparticulate delivery systems have been designed for targeted delivery of pharmaceuticals with improved half-life, bioavailability, and efficacy. Biodegradable polymer-based nanoparticles, nanoliposome, and dendrimers have attracted increasing interests for delivery of hydrophilic or hydrophobic drugs, genes, and macromolecules including the nucleic acids and proteins. Conjugation of the bioactive molecules with biocompatible and biodegradable polymeric matrix has been shown to provide sustained drug release along with the improved pharmacokinetics properties, therapeutic efficacy, and safety (40-42). These nanocarriers may provide an optimal drug concentration for a longer period of time that might result in the increased drug efficacy. In order to overcome the challenging issues associated with the earlier developed nanomaterials, research efforts have been attracted towards the development of the optimized nanocarriers including the lipid nanoparticles which protect the therapeutic agents against the enzymatic degradation and provide the controlled drug release from the lipid matrix and targeted delivery (43) leading to the improved pharmacological profile of drugs. In this respect, application of the nanostructured lipid carriers (NLCs), the promising carriers for poorly watersoluble drugs, have shown suitable characteristics including the long-term stability, biocompatibility, high drug loading capacity, and controlled drug release that may result in the reduced dosage frequency and side effects (44), hence, a better patient compliance in the case of the translation of basic research into the clinical practice. NLCs with large-scale production capability, may be loaded with a variety of therapeutic agents including those against the neurological disorders $(6,45,46)$.

Regarding the nanoformulations of FA, FA-loaded PolyD L lactide-co-glycolide (FA-PLGA) nanoparticles have been 
prepared by double emulsion method followed by the evaluation of the anticancer efficacy of the spherically-shaped nanoparticles in NCI-H460 cells. FA-PLGA nanoparticles enhanced DNA damage, intracellular reactive oxygen species (ROS) levels, and apoptotic morphological alterations in cancer cells. Furthermore, mitochondrial transmembrane potential was changed indicating the improved stability and bioavailability of FA at tumor site and enhanced cytotoxic activity of FA-PLGA (47). Electrospinning approach has also been used to prepare FA-encapsulated nanofibers with cytotoxic and antioxidant effects against HepG2 cells. These nanofibers have been represented as the promising therapeutic candidate against the liver cancer (48). Besides providing a high local drug concentration that might result in the enhanced tumor cell destruction, incorporation of FA into the nanofibers may also reduce the chemotherapeutic side effects (49). In this respect, electrospun nanofibers appear as promising candidates for tumor-targeted delivery of chemotherapeutic agents. Nanohybrids of FA and layered double hydroxide nanomaterials which have been prepared via the reconstruction and exfoliation-reassembly methods, have shown promising physicochemical properties for cellular uptake and enhances anticancer activity (50).

Using the ionotropic gelation method, FA-encapsulated chitosan nanoparticles have been synthesized followed by assessment of the stability and encapsulation of FA by thermal analyses, Fourier transform infrared spectrum, and nuclear magnetic resonance. Cytocompatiblity of the nanoparticles at effective concentrations has been shown in the human embryonic kidney (HEK-293) cell line indicating their suitable safety. These spherically-shaped and stable nanoparticles with positive zeta potential, a critical factor which facilitates the binding of nanoparticles to the negatively charged cell membrane leading to the disruption of their integrity and leakage of the intracellular material, have significantly reduced the cell metabolic activity of Candida albicans, an opportunistic fungal pathogen. In this context, FA-encapsulated chitosan nanoparticles have been represented as the effective alternative to the conventional antifungal therapeutics (51). For functional restoration following the traumatic spinal cord injury, self-assembled nanoparticles composed of FA and glycol chitosan (FA-GC) have been developed. Using these nanoparticles, the challenging issues regarding the pharmacokinetics and tissue distribution of free FA or GC have been overcome (52). Chitosan is mainly produced from the exoskeleton of crustaceans (e.g. shrimps and crabs) and similar to FA has shown anti-inflammatory, anti-oxidative, anti- excitotoxicity, and neuroprotective effects (53). Because of the poor solubility of chitosan, water-soluble glycol chitosan (GC) may be employed in which the neuroprotective effect of chitosan is preserved (54).

Following the chemical conjugation of FA to GC, the self-assembled nanoparticles composed of the hydrophobic FA core and hydrophilic GC shell with prolonged circulation time and distribution in white and gray matters (which are vulnerable to injury insults), FA and chitosan were successfully delivered to the injured site via the interrupted brain-spinal cord barriers and ruptured blood capillaries. In rats exposed to the spinal cord contusion injury, intravenous injection of nanoparticles has led to a remarkable recovery in the locomotor function at $2 \mathrm{~h}$ post injury. This, is of great therapeutic significance as following the primary spinal cord injury, protection of glial cells and neurons against the secondary degeneration is of critical importance. Indeed, targeted delivery of FA-GC nanoparticles into the injured gray matter in the spinal cord, prevents the progression of the pathological damages of glial cells and neurons at an early stage leading to protection of axons in the white matter and remarkable locomotor functional recovery. Treatment with methylprednisolone has been ineffective in this regard, hence, FA-GC nanoparticles appear as efficient neuroprotective agents in the spinal cord injuries. Histological improvement of the injured spinal cord including the suppressed the inflammatory response, cavity volume, and astrogliosis were also observed after treatment with FA-GC nanoparticles. These nanoparticles have also protected the primary neurons against the glutamate-induced excitotoxicity in vitro. Because of the restorative effects, FAGC nanoparticles have been represented as promising neuroprotective nanomedicine which may be applied in the clinical settings (55).

For oral administration of FA, microemulsion-based method has been used to prepare FA-loaded NLCs with small size distribution and sustained release profile. Gastrointestinal tract retention assay showed the efficiency of transport of the lipid nanoparticles from the stomach into the intestinal tract indicating the increased drug bioavailability. Furthermore, nanosized particles provided improved pharmacokinetic profile of FA as revealed by the enhanced drug solubility and absorption, and plasma concentrations (56).

Using electrospinning by which the ultrafine fibers or fibrous structures of various polymers with diameters in the range of nanometer or submicron may be fabricated (57), third generation solid dispersions of FA in composite nanofibers have been prepared. In vitro permeation and disso- 
lution tests revealed that nanofiber-based solid dispersions with much faster dissolution rate are able to rapidly release FA content and the permeation rate across the sublingual mucosa has been 13-fold higher than the control group (58). In this respect, development of the third generation solid dispersions by electrospinning appears as a promising approach to improve the pharmacological profile of the poorly water-soluble drugs. Indeed, electrospinning due to its unique properties, cost effectiveness, and ease of implementation is a popular nanotechnology approach for producing the nanofibers. Coaxial and side-by-side electrospinning as well as the multiple coaxial electrospinning are the promising techniques for development of the advanced nanofibers with well-defined microstructures and novel functions (59). For improved release profile of FA, a modified coaxial electrospinning process has been employed for fabrication of the functional polymer fibers with improved structure and performance. The prepared FA/zein composite fibers have shown high-quality in terms of the distribution, diameter, and sustained-release profile. In this context, the modified coaxial electrospinning process has been suggested as a useful tool to generate drug-encapsulated nanofibers with improved functional performance (60).

Pancreatic cancer is associated with a heterogeneous molecular pathogenesis including the genetic mutations and implication of a variety of oncogenic pathways, inflammation, and oxidative stress (61). Unfortunately, the current treatment options against the pancreatic carcinoma are usually ineffective (62). This has provoked increasing research efforts for development of the novel chemopreventive agents in order to delay or reverse the disease onset. For synergistic chemoprevention of the pancreatic cancer, combination of FA and the non-steroidal anti-inflammatory drug, aspirin, encapsulated in a novel chitosan-coated solid lipid nanoparticles drug delivery system has shown therapeutic potential against the disease as revealed by the reduced cell viability and increased apoptosis in the human pancreatic cancer cells (63). Noteworthy, chitosan facilitates the paracellular transport of drug molecules and mediates the opening of tight junctions between the neighboring epithelial cells that might result in the improved drug bioavailability (64). In pancreatic tumor xenograft mice model, application of FA and aspirin-loaded solid lipid nanoparticles with biodegradability, bioadhesivity, enhanced permeability properties, and prolonged residence time has led to the increased drug concentration at the site of absorption and significantly reduced the expression of proliferation proteins MKI67 and PCNA and increased the expression of apoptotic proteins including the p-ERK1/2, p-RB, and p21 indicating the pro-apoptotic effects of the nanoformulation. The anti-cancer effects might be due to the high antioxidant effect and free radical scavenging activity of FA and the modulatory effect of aspirin on the nuclear factor- $\mathrm{kB}(\mathrm{NF}-\mathrm{\kappa B})$, a transcription factor which participates in the induction of several genes for cytokines and is involved in a variety of physiological and pathological processes (65). Since the cloning of NF-kB genes and identification of NF-kB transcription factors, growing evidence has shown the critical role of NF-kB signaling pathways in the development and progression of various human cancers. In this respect, NF-kB signaling has been recognized as a target of cancer therapy $(66,67)$.

Application of the solid and liquid lipids as matrices for the preparation of nanoscaled lipid drug delivery systems has shown several advantages including the optimized drug properties such as the solubility, stability, and biocompatibility, reduced toxicity, controlled release profile, and industrial scalability (68). Nanoscaled lipid drug delivery systems may be used for systemic administration of the lipophilic drugs in a variety of diseases such as the central nervous system (CNS) disorders (69). Due to its remarkable antioxidant activity in Alzheimer's disease, FA has been entrapped into the lipid nanoparticles using the microemulsion technique. FAloaded nanoparticles with suitable physicochemical characteristics and cellular uptake have exhibited a significantly higher protective activity against the oxidative stress in neurons as compared to free FA (70). Using the same technique, trans-FA with anti-inflammatory, cardioprotective, and antioxidative effects has been encapsulated into the lipid nanoparticles for site-specific and controlled drug delivery and overcome the poor bioavailability of the compound. The prepared nanoformulation has shown a significantly improved drug release profile, stability during the storage, and pharmacokinetic parameters including the oral bioavailability (56).

Based on the protective effects of FA against the oxidative and nitrosative stress via up-regulating the key enhancers of the cell stress response such as the heme oxygenase/ biliverdin reductase pathway (71), FA has been loaded into the solid lipid nanoparticles in order to improve FA bioavailability (72). These spherically-shaped and biodegradable nanoparticles may be easily taken up by the brain due to their lipid nature, meanwhile, the size of nanoparticles should not exceed $200 \mathrm{~nm}$ (73). In order to bypass the blood-brain barrier, intranasal route may be applied which provides the possibility to deliver the drugs directly to the brain (74). In- 
tranasal administration of FA-loaded lipid nanoparticles has prevented the brain damage induced by free radicals (72) suggesting the therapeutic potential of this nanoformulation against the neurodegenerative disorders.

In human glioblastoma cancer U87MG cells, FA-NLCs with small particle size and high homogeneity have shown significantly higher effectiveness than the free compound (75). FA with antioxidant and anti-inflammatory properties is able to activate the apoptotic pathways in different types of cancers (76-78). Ferulic acid-NLC by inducing the cytotoxic effects via activating of the apoptotic pathway, appears as a promising therapeutic candidate against the glioblastoma, the most aggressive and common form of the brain tumor (79).

Application of the aerosolized FA-NLCs with controlled release pattern, slower clearance, improved pulmonary bioavailability and efficiency of FA, and reduced dosing frequency represents this inhalable nanoformulation as a promising therapeutic agent against the lung disorders (80). Application of the computational models for simulation of the fate of nanoparticles and evaluation of their interactions with the biological systems as well as predicting the side effects of drugs $(81,82)$ might result in the development of more efficient drug delivery systems.

\section{CONCLUSION}

Despite the promising therapeutic potentials of FA, application of this phytochemical in the clinical settings has remained challenging that might be due to its poor pharmacokinetic profile. Over the last decade, outstanding breakthroughs in nanotechnology have led to the development of biomaterials capable to interact with the biological systems as well as the highly-advanced nanovectors for targeted delivery of compounds with poor solubility or short half-lives. Designing the novel therapeutic agents with improved pharmacological profile such as the nanoformulations of FA may provide better prospects for treatment of a variety of disorders. Successful translation of the basic findings into the clinical settings may facilitate obtaining the regulatory approvals and commercialization of products.

Conflict of interest: the authors declare no conflict of interest.

\section{REFERENCES}

1. Tapsell LC, Hemphill I, Cobiac L, Patch CS, Sullivan DR, Fenech M, et al. Health benefits of herbs and spic- es: the past, the present, the future. Med J Aust 2006; 185: S4-S24. PMD: 17022438.

2. Gohil KJ, Kshirsagar SB, Sahane RS. Ferulic acidA comprehensive pharmacology of an important bioflavonoid. Int J Pharm Sci Res 2012; 3: 700-710. DOI:10.13040/IJPSR.0975-8232.3(3).700-10.

3. Yan JJ, Cho JY, Kim HS, Kim KL, Jung JS, Huh SO, et al. Protection against b-amyloid peptide toxicity in vivo with long-term administration of ferulic acid. Br J Pharmacol 2001; 133: 89-96. DOI:10.1038/ sj.bjp.0704047.

4. Hassanzadeh P, Arbabi E, Atyabi F, Dinarvand R. Ferulic acid exhibits antiepileptogenic effect and prevents oxidative stress and cognitive impairment in the kindling model of epilepsy. Life Sci 2017; 179: 9-14. DOI: 10.1016/j.1fs.2016.08.011.

5. Cheng CY, Ho TY, Lee EJ, Su SY, Tang NY, Hsieh CL. Ferulic acid reduces cerebral infarct through its antioxidative and anti-inflammatory effects following transient focal cerebral ischemia in rats. Am J Chin Med 2008; 36: 1105-1119. DOI:10.1142/S0192415X08006570.

6. Hassanzadeh P, Arbabi E, Atyabi F, Dinarvand R. Ferulic acid-loaded nanostructured lipid carriers: A promising nanoformulation against the ischemic neural injuries. Life Sci 2017; 28. pii: S0024-3205(17)30623-9. DOI: 10.1016/j.lfs.2017.11.046.

7. Ou S, Kwok KC. Ferulic acid: pharmaceutical functions, preparation and applications in foods. J Sci Food Agric 2004; 84: 1261-1269. doi:10.1002/jsfa.1873.

8. Brenelli de Paiva L, Goldbeck R, Dantas dos Santos W, Marcio Squina F. Ferulic acid and derivatives: molecules with potential application in the pharmaceutical field. Braz J Pharm Sci 2013; 49: 395-411. http:// dx.doi.org/10.1590/S1984-82502013000300002.

9. Sudheer AR, Muthukumaran S, Kalpana C, Srinivasan M, Menon VP. Protective effect of ferulic acid on nicotine-induced DNA damage and cellular changes in cultured rat peripheral blood lymphocytes: a comparison with Nacetylcysteine. Toxicol In Vitro 2007; 21: 576585. DOI:10.1016/j.tiv.2006.11.006.

10. Liu Y. Pharmaceutical composition for increasing immunity and decreasing side effects of anticancer chemotherapy. US patent 1987; 4,687,761.

11. Wang B, Ouyang J, Liu Y, Yang J, Wei L, Li K, Yang H. Sodium ferulate inhibits atherosclerogenesis in hyperlipidemia rabbits. J Cardiovasc Pharmacol 2004; 43: 549-554. PMID:15085066. 
12. Mathew S, Abraham TE. Bioconversions of ferulic acid, an hydroxycinnamic acid. Crit Rev Microbiol 2006; 32: 115-125. DOI:10.1080/10408410600709628.

13. Lin FH, Lin JY, Gupta RD, Tournas JA, Burch JA, Se$\lim$ MA, et al. Ferulic acid stabilizes a solution of vitamins $\mathrm{C}$ and $\mathrm{E}$ and doubles its photoprotection of skin. J Invest Dermatol 2005; 125: 826-832. DOI:10.1111/ j.0022-202X.2005.23768.x.

14. Lina CM, Chiuc JH, Wu IH, Wang BW, Pan CM, Cheng YH. Ferulic acid augments angiogenesis via VEGF, PDGF and HIF-1a. J Nutr Biochem 2010; 21: 627-633. doi: 10.1016/j.jnutbio.2009.04.001.

15. Hassanzadeh $\mathrm{P}$, Arbabi E, Atyabi F, Dinarvand R. Ferulic acid-loaded nanostructured lipid carriers: A promising nanoformulation against the ischemic neural injuries. Life Sci 2016; 3205: 30623-30629. DOI: 10.1016/j.lfs.2017.11.046.

16. Lenzi J, Rodrigues AF, de Sousa Rós A, Blanski de Castro B, de Lima D, Delwing Dal Magro D. Ferulic acid chronic treatment exerts antidepressant-like effect: role of antioxidant defense system. Metab Brain Dis 2015; 30: 1453-1463. DOI 10.1007/s11011-015-97256.

17. Zeni ALB, Zomkowski ADE, Maraschin M, Rodrigues ALS, Tasca CI. Involvement of PKA, CaMKII, PKC, MAPK/ERK and PI3K in the acute antidepressant-like effect of ferulic acid in the tail suspension test. Pharmacol Biochem Behav 2012; 103: 181-186. DOI:10.1016/j.pbb.2012.08.020.

18. Castren E, Voikar V, Rantamaki T. Role of neurotrophic factors in depression. Curr Opin Pharmacol 2007; 7: 18-21. DOI 10.1016/j.coph.2006.08.009.

19. Aloe L, Rocco ML, Balzamino BO, Micera A. Nerve growth factor: a focus on neuroscience and therapy. Curr Neuropharmacol 2015; 13: 294-303. PMCID:PMC4812798.

20. Berton O, Nestler EJ. New approaches to antidepressant drug discovery: Beyond monoamines. Nat Rev Neurosci 2006; 7: 137-151. DOI:10.1038/nrn 1846.

21. Levi-Montalcini R. The nerve growth factor and the neuroscience chess board. Prog Brain Res 2004; 146: 525-527. PMD: 14699984.

22. Lad SP, Neet KE, Mufson EJ. Nerve growth factor: structure, function and therapeutic implications for Alzheimer's disease. Curr Drug Targets CNS Neurol Disord 2003; 2: 315-34. PMID:14529363.

23. Hassanzadeh P, Hassanzadeh A. The CB1 receptor-me- diated endocannabinoid signaling and NGF: the novel targets of curcumin. Neurochem Res 2012; 37: 11121120. DOI 10.1007/s11064-012-0716-2.

24. Hassanzadeh P, Rahimpour S. The cannabinergic system is implicated in the upregulation of central NGF protein by psychotropic drugs. Psychopharmacology 2011; 215: 129-141. DOI 10.1007/s00213-010-2120-4.

25. Hassanzadeh $\mathrm{P}$, Hassanzadeh A. Implication of NGF and endocannabinoid signaling in the mechanism of action of sesamol: a multi-target natural compound with therapeutic potential. Psychopharmacology 2013; 229: 571-578. DOI 10.1007/s00213-013-3111-z.

26. Hassanzadeh P, Arbabi E, Atyabi F, Dinarvand R. The endocannabinoid system and NGF are involved in the mechanism of action of resveratrol: a multi-target nutraceutical with therapeutic potential in neuropsychiatric disorders. Psychopharmacology 2016; 233: 10871096. DOI 10.1007/s00213-015-4188-3.

27. Hassanzadeh P, Hassanzadeh A. Involvement of the neurotrophin and cannabinoid systems in the mechanisms of action of neurokinin receptor antagonists. Eur Neuropsychopharm 2011; 21: 905-917. DOI:10.1016/j. euroneuro.2011.01.002.

28. Hassanzadeh P, Hassanzadeh A. Effects of different psychotropic agents on the central nerve growth factor protein. Iran J Basic Med Sci 2010; 13: 202-209. DOI: 10.22038/ijbms.2010.5064.

29. Hassanzadeh P, Arbabi E, Atyabi F, Dinarvand R. Ferulic acid, a phenolic compound with therapeutic effects in neuropsychiatric disorders, stimulates the production of NGF and endocannabinoids in rat brain. Physiol Pharmacol 2017 (in press) URL: http://phypha.ir/ppj/ article-1-1265-en.html.

30. Banasr M, Valentine GW, Li XY, Gourley SL, Taylor JR, Duman RS. Chronic unpredictable stress decreases cell proliferation in the cerebral cortex of the adult rat. Biol Psychiatry 2007; 62: 496-504. DOI:10.1016/j.biopsych.2007.02.006.

31. Conner JM, Franks KM, Titterness AK, Russell K, Merrill DA, Christie BR et al. NGF is essential for hippocampal plasticity and learning. J Neurosci 2009; 29: 10883-10889. PMID:19726646.

32. Moises HC, Womble MD, Washburn MS, Williams LR. Nerve growth factor facilitates cholinergic neurotransmission between nucleus basalis and the amygdala in rat: an electrophysiological analysis. J Neurosci 1995; 15: 8131-8142. PMID:8613748. 
33. Miwa T, Moriizumi T, Horikawa I, Uramoto N, Ishimaru T, Nishimura T, et al. Role of nerve growth factor in the olfactory system. Microsc Res Tech 2002; 58: 197-203. DOI:10.1002/jemt.10149.

34. Hassanzadeh P. The edocannabinoid system: critical for the neuroptrophic action of psychotropic drugs. Biomed Rev 2010; 21: 31-46. ISSN 1314-1929.

35. Hassanzadeh P. Discovery of the endocannabinoid system: A breakthrough in neuroscience. Arch Neurosci 2014; 2: e15030. DOI: 10.5812/archneurosci.15030.

36. Hassanzadeh P, Hassanzadeh A. The role of the endocannabinoids in suppression of the hypothalamic-pituitary-adrenal axis activity by doxepin. Iran J Basic Med Sci 2011; 14: 414-421. PMCID: PMC3586843.

37. Viveros MP, Marco EM, File SE. Endocannabinoid system and stress and anxiety responses. Pharmacol Biochem Behav 2005; 81: 331-342. DOI:10.1016/j. pbb.2005.01.029

38. Bambico FR, Duranti A, Tontini A, Tarzia G, Gobbi G. Endocannabinoids in the treatment of mood disorders: evidence from animal models. Curr Pharm Des 2009; 15: 1623-1646. PMID:19442178.

39. Zhaohui Zhao, Mohammed H. Moghadasian. Chemistry, natural sources, dietary intake and pharmacokinetic properties of ferulic acid: A review. Food Chem 2008; 109: 691-702. doi:10.1016/j.foodchem.2008.02.039.

40. Patel T, Zhou J, Piepmeier JM, Saltzman WM. Polymeric nanoparticles for drug delivery to the central nervous system. Adv Drug Deliv Rev 2012; 64: 701705. DOI: 10.1016/j.addr.2011.12.006.

41. Fernandes R, Gracias DH. Self-folding polymeric containers for encapsulation and delivery of drugs. $A d v$ Drug Deliv Rev 2012; 64: 1579-1589. DOI: 10.1016/j. addr.2012.02.012.

42. Sun NF, Meng QY, Tian AL, Hu SY, Wang RH, Liu ZX, et al. Nanoliposome mediated FL/TRAIL double-gene therapy for colon cancer: in vitro and in vivo evaluation. Cancer Lett 2012; 315: 69-77. DOI:10.1016/j. canlet.2011.10.010.

43. Joshi MD, Müller RH. Lipid nanoparticles for parenteral delivery of actives. Eur J Pharm Biopharm 2009; 71: 161-172. DOI: 10.1016/j.ejpb.2008.09.003.

44. Kumbhar DD, Pokharkar VB. Engineering of a nanostructured lipid carrier for the poorly water-soluble drug, bicalutamide: Physicochemical investigations. Colloids Surf A Physicochem Eng Asp 2013; 416: 3242. https://doi.org/10.1016/j.colsurfa.2012.10.031.
45. Hassanzadeh P, Atyabi F, Dinarvand R, Dehpour AR, Azhdarzadeh M, Dinarvand M. Application of nanostructured lipid carriers: The prolonged protective effects for sesamol in in vitro and in vivo models of ischemic stroke via activation of PI3K signalling pathway. Daru Int J Pharm 2017; (in press).

46. Hassanzadeh P. Nanopharmaceuticals: Innovative theranostics for the neurological disorders. Biomed Rev 2014; 25: 25-34. DOI: 10.14748/bmr.v25.1043.

47. Jose Merlin JP, Rajendra Prasad N, Shibli SMA, Sebeela M . Ferulic acid loaded Poly-D L lactide-co-glycolide nanoparticles: Systematic study of particle size, drug encapsulation efficiency and anticancer effect in nonsmall cell lung carcinoma cell line in vitro. Biomed Prevent Nutr 2012; 2: 69-76. http://dx.doi.org/10.1016/j. bionut.2011.12.007.

48. Vashisth P, Kumar N, Sharma M, Pruthi V. Biomedical applications of ferulic acid encapsulated electrospun nanofibers. Biotech Rep 2015: 8: 36-44. http://dx.doi. org/10.1016/j.btre.2015.08.008.

49. Vashisth P, Sharma M, Nikhil K, Singh H, Panwar R, Pruthi PA, Pruthi V. Antiproliferative activity of ferulic acid-encapsulated electrospun PLGA/PEO nanofibers against MCF-7 human breast carcinoma cells. Biotechnology 2015; 5: 303-315. DOI 10.1007/s13205-0140229-6.

50. Kim HJ, Ryu K, Kang JH, Choi AJ, Kim T, Oh JM. Anticancer activity of ferulic acid-inorganic nanohybrids synthesized via two different hybridization routes, reconstruction and exfoliation-reassembly. Sci World J 2013; 2013: Article ID 421967. http://dx.doi. org/10.1155/2013/421967.

51. Panwar R, Pemmaraju SC, Sharma AK, Pruthi V. Efficacy of ferulic acid encapsulated chitosan nanoparticles against Candida albicans biofilm. Microb Pathogen 2016; 95: 21 e31. DOI: 10.1016/j.micpath.2016.02.007.

52. Wu W, Lee SY, Wu X, Tyler JY, Wang H, Ouyang Z, et al. Neuroprotective ferulic acid (FA)eglycol chitosan (GC) nanoparticles for functional restoration of traumatically injured spinal cord. Biomaterials 2014; 35: 2355e2364. DOI:10.1016/j.biomaterials.2013.11.074.

53. Ratih P, Kim SK. Neuroprotective properties of chitosan and its derivatives. Marine Drugs 2010; 8: $2117-$ 2128. [PubMed: 20714426].

54. Na JH, Lee SY, Lee S, Koo H, Min KH, Jeong SY, Yuk $\mathrm{SH}$, et al. Effect of the stability and deformability of self assembled glycol chitosan nanoparticles on tumor- 
targeting efficiency. J Control Release 2012; 163: 2-9. [PubMed: 22846988].

55. Wu W, Lee SY, Wu X, Tyler JY, Wang H, Ouyang Z, et al. Neuroprotective ferulic acid (FA)-glycol chitosan (GC) nanoparticles for functional restoration of traumatically injured spinal cord. Biomaterials 2014; 35: 2355-2364. DOI: 10.1016/j.biomaterials.2013.11.074.

56. Zhang Y, Li Z, Zhang K, Yang G, Wang Z, Zhao J, et al. Ethyl oleate-containing nanostructured lipid carriers improve oral bioavailability of trans-ferulic acid ascompared with conventional solid lipid nanoparticles. Int J Pharm 2016; 511: 57-64. DOI: 10.1016/j. ijpharm.2016.06.131.

57. Dzenis Y. Spinning continuous fibres for nanotechnology. Science 2004; 304: 1917-1919. DOI: 10.1126/science. 1099074

58. Yua DG, Yangb JM, Branford-Whitec C, Lub P, Zhangb L, Zhu LM. Third generation solid dispersions of ferulic acid in electrospun composite nanofibers. Int J Pharm 2010; 400: 158-164. DOI:10.1016/j.ijpharm.2010.08.010.

59. Wu XM, Branford-White C, Yu DG, Chatterton NP, Zhu LM. Preparation of core-shell PAN nanofibers encapsulated $\alpha$-tocopherol acetate and ascorbic acid 2-phosphate for photoprotection. Colloids Surf B: Biointerfaces 2011; 82: 247-252. DOI: 10.1016/j.colsurfb.2010.08.049.

60. Yanga JM, Zha LS, Yub DG, Liu J. Coaxial electrospinning with acetic acid for preparing ferulic acid/zein composite fibers with improved drug release profiles. Colloids Surf B: Biointerfaces 2013; 102: 737- 743. DOI: 10.1016/j.colsurfb.2012.09.039.

61. Garcea G, Dennison AR, Steward WP, Berry DP. Role of inflammation in pancreatic carcinogenesis and the implications for future therapy. Pancreatology 2005; 5: 514-529. DOI:10.1159/000087493.

62. DeSantis CE, Lin CC, Mariotto AB, Siegel RL, Stein $\mathrm{KD}$, Kramer JL, et al. Cancer treatment and survivorship statistics. Cancer J Clin 2014; 64: 252-271. DOI: 10.3322/caac. 21235 .

63. Thakkar A, Chenreddy S, Wang J, Prabhu S. Ferulic acid combined with aspirin demonstrates chemopreventive potential towards pancreatic cancer when delivered using chitosan-coated solid-lipid nanoparticles. Cell Biosci 2015; 5: 46. DOI 10.1186/s13578-0150041-y.

64. Fonte P, Andrade F, Araujo F, Andrade C, Neves J, Sar- mento B. Chitosan coated solid lipid nanoparticles for insulin delivery. Methods Enzymol 2012; 508: 295-314. DOI: 10.1016/B978-0-12-391860-4.00015-X.

65. Agrawal A, Cha-Molstad H, Samols D, Kushner I. Overexpressed nuclear factor- $\mathrm{\kappa B}$ can participate in endogenous $\mathrm{C}$-reactive protein induction, and enhances the effects of C/EBP $\beta$ and signal transducer and activator of transcription-3. Immunology 2003; 108: 539547. PMCID:PMC1782914.

66. Kim HJ, Hawke N, Baldwin AS. NF-kappaB and IKK as therapeutic targets in cancer. Cell Death Differ 2006; 13: 738-747. DOI:10.1038/sj.cdd.4401877.

67. Hassanzadeh P. Colorectal cancer and NF- $\kappa \mathrm{B}$ signaling pathway. Gastroenterol Hepatol Bed Bench 2011; 4: 127-132. PMID:24834170.

68. Müller RH, Shegokar R, Keck CM. 20 Years of lipid nanoparticles (SLN \&NLC): present state of development and industrial application. Curr Drug Discov Technol 2011; 8: 207-227. PMID:21291409.

69. Martins S, Tho I, Reimold I, Fricker G, Souto E, Ferreira $\mathrm{D}$, Brandl M. Brain delivery of camptothecin by means of solid lipid nanoparticles: formulation design, in vitro and in vivo studies. Int J Pharm 2012; 439: 4962. DOI: 10.1016/j.jpharm.2012.09.054.

70. Bondi M, Montana G, Craparo E, Picone P, Capuano $\mathrm{G}$, Carlo MD, et al. Ferulic acid-loaded lipid nanostructures as drug delivery systems for Alzheimer's disease: Preparation, characterization and cytotoxicity studies. Curr Nanosci 2009; 5: 26-32. DOI: 10.2174/157341309787314656.

71. Fetoni AR, Mancuso C, Eramo SL, Ralli M, Piacentini $\mathrm{R}$, Barone $\mathrm{E}$, et al. In vivo protective effect of ferulic acid against noise-induced hearing loss in the guinea-pig. Neuroscience 2010; 169: 1575-1588. doi: 10.1016/j.neuroscience.2010.06.02.

72. Trombino S, Cassano R, Ferrarelli T, Barone E, Picci N, Mancuso C. Trans-ferulic acid-based solid lipid nanoparticles and their antioxidant effect in rat brain microsomes. Colloids Surf B Biointerfaces 2013; 109: 273-279. DOI: 10.1016/j.colsurfb.2013.04.005.

73. Kaur IP, Bhandari R, Bhandari S, Kakkar V. Potential of solid lipid nanoparticles in brain targeting. $J$ Control Release 2008; 127: 97-109. DOI: 10.1016/j.jconrel.2007.12.018.

74. Dhuria SV, Hanson LR, Frey WH 2nd. Intranasal delivery to the central nervous system: mechanisms and experimental considerations. J Pharm Sci 2010; 99: 
1654-1673. DOI: 10.1002/jps.21924.

75. Carbone C, Campisi A, Musumeci T, Raciti G, Bonfanti R, Puglisi G. FA-loaded lipid drug delivery systems: Preparation, characterization and biological studies. Eur J Pharm Sci 2014; 52:12-20. http://dx.doi. org/10.1016/j.ejps.2013.10.003.

76. Serafim TL, Carvalho FS, Marques MP, Calheiros R, Silva T, Garrido J, et al. Lipophilic caffeic and ferulic acid derivatives presenting cytotoxicity against human breast cancer cells. Chem Res Toxicol 2011; 24: 763774. DOI: $10.1021 / \mathrm{tx} 200126 \mathrm{r}$.

77. Karthikeyan S, Kanimozhi G, Prasad NR, Mahalakshmi R. Radiosensitizing effect of ferulic acid on human cervical carcinoma cells in vitro. Toxicol In Vitro 2011; 25: 1366-1375. DOI: 10.1016/j.tiv.2011.05.007.

78. Bandugula VR, Rajendra Prasad N. 2-Deoxy-d-glucose and ferulic acid modulates radiation response signaling in non-small cell lung cancer cells. Tumour Biol 2013; 34: 251-259. DOI: 10.1007/s13277-012-0545-6.
79. Van Meir EG, Hadjipanayis CG, Norden AD, Shu HK, Wen PY, Olson JJ. Exciting new advantages in neurooncology: the avenue to a cure for malignant glioma. Cancer J Clin 2010; 60:166-193. DOI: 10.3322/ caac.20069.

80. Hassanzadeh P, Arbabi E, Rostami F, Atyabi F, Dinarvand R. Aerosol delivery of ferulic acid-loaded nanostructured lipid carriers: A promising therapeutic approach against the lung disorders. Physiol Pharmacol 2017 (in press) URL: http://phypha.ir/ppj/article1-1295-en.html.

81. Hassanzadeh P. Computational modelling: Moonlighting on the neuroscience and medicine. Biomed Rev 2013; 24: 25-31. DOI: 10.14748/bmr.v24.19.

82. Hassanzadeh P, Atyabi F, Dinarvand R. Application of modelling and nanotechnology-based approaches: The emergence of breakthroughs in theranostics of central nervous system disorders. Life Sci 2017; 182: 93-103. DOI: 10.1016/j.lfs.2017.06.001. 